Portland State University

PDXScholar

\title{
Frequency Based Noise Coherence-Function Extension and Application to Passive Bottom-Loss Estimation
}

\author{
Lanfranco Muzi \\ Portland State University, lan.muzi@gmail.com \\ Martin Siderius \\ Portland State University, siderius@pdx.edu \\ Peter L. Nielsen \\ Center for Maritime Research and Experimentation
}

Follow this and additional works at: https://pdxscholar.library.pdx.edu/ece_fac

Part of the Acoustics, Dynamics, and Controls Commons, and the Electrical and Computer Engineering Commons

Let us know how access to this document benefits you.

\section{Citation Details}

Muzi, L., Siderius, M., \& Nielsen, P. L. (2016). Frequency based noise coherence-function extension and application to passive bottom-loss estimation. The Journal of the Acoustical Society of America, 140(3), 1513-1524.

This Article is brought to you for free and open access. It has been accepted for inclusion in Electrical and Computer Engineering Faculty Publications and Presentations by an authorized administrator of PDXScholar. Please contact us if we can make this document more accessible: pdxscholar@pdx.edu. 


\title{
Frequency based noise coherence-function extension and application to passive bottom-loss estimation
}

\author{
Lanfranco Muzia) and Martin Siderius \\ Department of Electrical and Computer Engineering, Portland State University, 1900 SW 4th Avenue, \\ Portland, Oregon 97201, USA \\ Peter L. Nielsen \\ Centre for Maritime Research and Experimentation (CMRE), NATO Science and Technology Organization, \\ Viale S. Bartolomeo 400, La Spezia, 19126, Italy
}

(Received 29 October 2015; revised 30 July 2016; accepted 10 August 2016; published online 7 September 2016)

Accurate modeling of acoustic propagation in the ocean waveguide is important to SONARperformance prediction. Particularly in shallow waters, a crucial contribution to the total transmission loss is the bottom refection loss, which can be estimated passively by beamforming the natural surface-noise acoustic field recorded by a vertical line array of hydrophones. However, the performance in this task of arrays below $2 \mathrm{~m}$ of length is problematic for frequencies below $10 \mathrm{kHz}$ It is shown in this paper that, when the data are free of interference from sources other than wind and wave surface noise, data from a shorter array can be used to approximate the coherence function of a longer array. This improves the angular resolution of the estimated bottom loss, often making use of data at frequencies above the array design frequency. Application to simulated and experimental data shows that the technique, rigorously justified for a halfspace bottom, is effective also on more complex bottom types. Dispensing with active sources, small autonomous underwater vehicles equipped with short arrays can be envisioned as compact, efficient seabed-characterization systems. The proposed technique is shown to improve significantly the reflection-loss estimate of an array that would be a candidate for such application. (C) 2016 Acoustical Society of America.

[http://dx.doi.org/10.1121/1.4962229]

[SED]

Pages: $1513-1524$

\section{INTRODUCTION}

The prediction of SONAR performance depends upon the accuracy of acoustic propagation modeling in the ocean waveguide. This in turn requires the characterization of the acoustic properties of the water column and the boundaries, i.e., the surface and the bottom, with the latter being of crucial importance in shallow-water environments. This paper focuses in particular on determining the seabed reflection loss (hereafter shortly referred to as "bottom loss," or "BL"), a significant contributor to the transmission loss, as a function of frequency and grazing angle. The thickness and physical properties of the bottom layers (which can vary dramatically within a few hundred meters of distance) $)^{1,2}$ determine the dependence of the bottom loss on these two variables. Direct in situ measurement of the bottom properties (e.g., by collection and analysis of seabed cores) is costly and difficult. ${ }^{3,4}$ Data for propagation models are, therefore, typically obtained either from existing environmental databases, or by geoacoustic inversion of measured acoustic data. While the first source has been shown to be often unreliable for SONAR prediction, ${ }^{5}$ geoacoustic inversion is potentially capable of providing adequate resolution in space for accurate propagation modeling. In what is perhaps its most widely employed methodology, the technique

${ }^{\text {a)} E l e c t r o n i c ~ m a i l: ~ m u z i @ p d x . e d u ~}$ uses acoustic sources (sound projectors, explosive charges, or even sources of opportunity, such as ship noise) and hydrophone arrays to measure the acoustic field, and modelbased matched-field processing to determine the seabed properties. $^{6-8}$

Alternatively, a passive technique was proposed by Harrison and Simons, based on beamforming of the acoustic field produced by noise naturally occurring at the surface (generated by wind, breaking waves, and rain) recorded by a vertical line array (VLA) deployed in the water column. ${ }^{9}$ By eliminating the need for artificial acoustic sources, the technique achieves several benefits, among which reduced equipment complexity, cost, weight, and power consumption. Harrison and Simons' technique has proven effective in the $100-5000 \mathrm{~Hz}$ frequency range, when employing arrays of lengths between a few meters and several tens of meters. However, when the array length falls below $2 \mathrm{~m}$, at the frequency range indicated above, the inherently poor angular resolution of the beams becomes a matter of concern for the quality of the estimated bottom loss, causing an underestimation of the loss and poor resolution of its grazing-angle dependent features. ${ }^{10}$

More recently, a study has provided a detailed proof (based on a model proposed by Harrison) of the technique, and shown that the angular resolution of the BL estimated from array data can be improved by exploiting specific properties of the ambient-noise vertical coherence function to remove some undesirable effects of conventional beamforming. ${ }^{11,12}$ Another 
recent study has proposed to employ existing algorithms for the extrapolation of band-limited signals, to reconstruct the noise coherence function of a longer array starting from data measured by a shorter array. The extrapolation results appeared promising, but the potential of this technique for bottom-loss estimation has not been investigated yet. ${ }^{13}$

In this paper, the idea of overcoming the limitations of short arrays by synthesizing the coherence function of a longer array is treated with the specific purpose of improving the performance of bottom-loss estimation (particularly the angular resolution) through Harrison and Simons' technique. However, instead of applying extrapolation algorithms, the proposed technique uses data measured at different frequencies by the physical hydrophones, to approximate the coherence function at the location of the sensors of a longer array. The technique is computationally simple, and makes a more efficient use of the frequency bandwidth available to modern acquisition systems, which often extends well beyond the array design frequency.

An attractive field of application of short arrays is autonomous-underwater-vehicle (AUV) based bottom survey. Dispensing with the use of active sources makes it now possible to envision an efficient, cost effective survey tool for seabed characterization, composed only of a short array and acquisition system mounted on a small AUV, such as the eFolaga employed in a preliminary feasibility study of such a system. ${ }^{14}$ For AUVs of this class, constraints related to weight, drag, and power consumption would reasonably require array lengths below $2 \mathrm{~m}$. While an ambient-noise data set acquired by an AUV and suitable for this study is not currently available, this paper includes an application of the proposed algorithm to a data set recorded by an array whose features (eight elements, $1.26 \mathrm{~m}$ length) would make it a good candidate for AUV deployment.

The rest of this paper is organized as follows: Section II describes Harrison and Simons' technique for passive bottom-loss estimation, shows the role played by the noise spatial coherence function in the process, and illustrates the dependence of the function on the signal frequency and array sensor spacing. Section III describes how frequency based extension of the coherence function is implemented. Section IV uses simulation to introduce the application of this technique to passive bottom-loss estimation. Section V shows the results of the technique on measured data from several experimental campaigns, and Sec. VI summarizes the main findings of this study.

\section{PASSIVE BOTTOM-LOSS ESTIMATION AND THE NOISE SPATIAL COHERENCE FUNCTION}

\section{A. Bottom-loss estimation and beamforming}

Given a plane wave front of angular frequency $\omega$ incident upon the bottom at grazing angle $\theta_{b}>0$ (see Fig. 1 for the definition of all geometric quantities), the bottom loss is defined as ${ }^{1}$

$$
\operatorname{BL}\left(\theta_{b}, \omega\right)=-10 \log _{10} R\left(\theta_{b}, \omega\right),
$$

where $R\left(\theta_{b}, \omega\right)$ is the plane-wave power reflection coefficient of the bottom. Harrison and Simons ${ }^{9}$ have proposed a

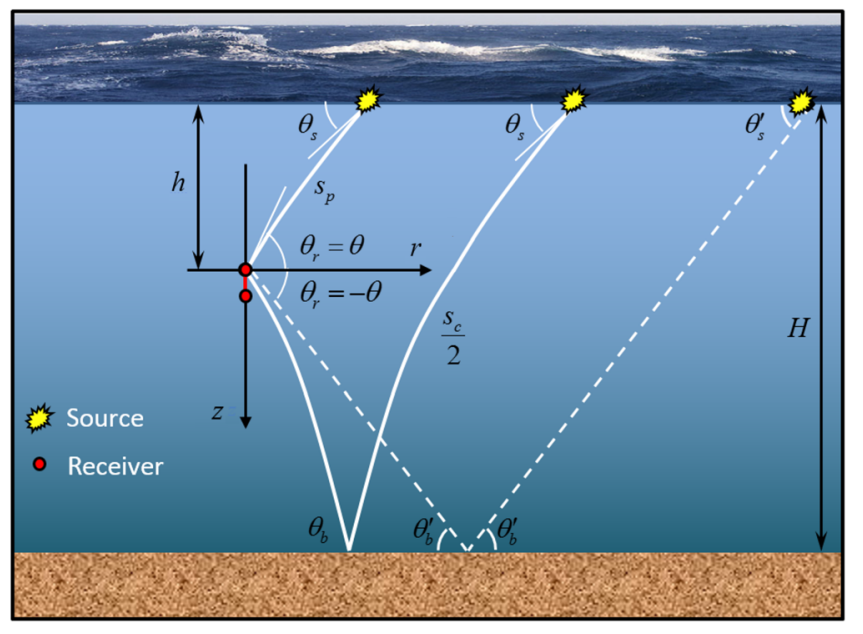

FIG. 1. (Color online) Definition of coordinate system and geometric quantities. For constant sound speed, the rays are straight lines (dashed), and $\theta_{s}^{\prime}=\theta_{b}^{\prime}=\theta_{r}=\theta$. The thick solid lines represent ray paths in the presence of a sound-speed profile. The same angle at the receiver $\theta_{r}$ is considered in both cases.

passive technique for estimating the bottom loss that has proven effective in several studies; ${ }^{10,15-17}$ the details of the technique have already been described in literature, ${ }^{9,11}$ but it is convenient to summarize here the parts that are relevant to this work.

In this technique, a vertical line array of hydrophones is deployed in the water column to sample the marine ambientnoise field at discrete locations in space. The measured data are then beamformed to obtain an estimate $\hat{B}(\vartheta, \omega)$ of the average beam power impinging on the array at a given steering angle $\vartheta$. In this paper, the angle $\vartheta=0$ corresponds to the array being steered towards broadside (i.e., horizontally, for a vertical array), $\vartheta>0$ towards the surface, and $\vartheta<0$ towards the bottom. $\hat{B}(\vartheta, \omega)$ is estimated via beamforming at opposite angles with respect to the horizontal, and the ratio of these measurements gives an estimate $\hat{R}\left(\theta_{b}, \omega\right)$ of the power reflection coefficient:

$$
\hat{R}\left(\theta_{b}, \omega\right)=\frac{\hat{B}(-\theta, \omega)}{\hat{B}(\theta, \omega)},
$$

where $\theta=\left|\theta_{r}\right|$ is the absolute value of the angle at the receiver $\theta_{r}$ (i.e., the angle at which a ray reaches the receiver) corresponding to the angle at the bottom $\theta_{b} . \hat{R}\left(\theta_{b}, \omega\right)$ is then used in Eq. (1) to estimate the bottom loss.

The beamforming operation can be mathematically formalized as a matrix product (for the sake of simplicity, in the remainder of this paper the dependence on frequency and angle will be dropped on the right hand side of equations):

$$
\hat{B}(\vartheta, \omega)=\mathbf{w}^{H} \hat{\mathbf{C}}_{\omega} \mathbf{w}
$$

where $\mathbf{w}(\vartheta, \omega)=\left[w_{1}, w_{2}, \ldots, w_{M}\right]^{T}$ is the weight vector $(T$ denotes the transpose operation) that achieves the spatial filtering in direction $\vartheta$, and $H$ indicates the conjugate transpose operation. Harrison and Simons' technique makes use of the "conventional beamformer" (CBF), for which the weight of the $m$ th element in the array is computed as 


$$
w_{m}(\vartheta, \omega)=\mathrm{s}_{\mathrm{m}} e^{-i(m-1) \frac{\omega}{c} d \sin \vartheta}
$$

where $c$ is the sound speed at the receivers, $d$ is the array inter-element spacing (assumed constant throughout the array), and $s_{m}$ is a shading coefficient (equal to 1 if no shading is applied to the array).

The symbol $\hat{\mathbf{C}}_{\omega}$ in Eq. (3) represents an estimate of the "cross-spectral-density matrix" $\mathbf{C}_{\omega}$ (hereafter also referred to as "CSD matrix"), obtained by averaging the outer product $\mathbf{p}_{k}(\omega) \mathbf{p}_{k}^{H}(\omega)$ over $K$ data segments:

$$
\hat{\mathbf{C}}_{\omega}=\frac{1}{K} \sum_{k=1}^{K} \mathbf{p}_{k} \mathbf{p}_{k}^{H} .
$$

The vector $\mathbf{p}(\omega)=\left[p_{1}(\omega), p_{2}(\omega), \ldots, p_{M}(\omega)\right]^{T}$, where $p_{m}(\omega)$ $=p\left(\mathbf{r}_{m}, \omega\right)=\mathscr{f}\left\{p_{m}(t)\right\}$, represents the data from the $m$ th hydrophone in the array $(\mathscr{f}\{\cdot\}$ denotes the Fourier transform). The symbol $p_{m}(t)$ is a simplified notation for $p\left(\mathbf{r}_{m}, t\right)$, designating the time series of the pressure field at the position $\mathbf{r}_{m}$, where the $m$ th hydrophone is located.

\section{B. Normalized and unnormalized spatial coherence function}

The performance of an array of sensors in noise depends upon the accuracy of the estimate of the CSD matrix-i.e., the second order statistics of the noise field at the sensors. This is modeled in physics by the spatial coherence function $C_{\omega}\left(\mathbf{r}_{1}, \mathbf{r}_{2}\right)$ of the pressure field $p(\mathbf{r}, t)$, defined between two points (i.e., sensor locations) in space $\mathbf{r}_{1}$ and $\mathbf{r}_{2}$, in its unnormalized form, as the ensemble average of the product $p\left(\mathbf{r}_{1}, \omega\right) p^{*}\left(\mathbf{r}_{2}, \omega\right)$ :

$$
C_{\omega}\left(\mathbf{r}_{1}, \mathbf{r}_{2}\right) \equiv\left\langle p\left(\mathbf{r}_{1}, \omega\right) p^{*}\left(\mathbf{r}_{2}, \omega\right)\right\rangle,
$$

where $*$ indicates complex conjugate. The explicit link with beamforming is established by noting that element $(i, j)$ in $\mathbf{C}_{\omega}$ is given by $C_{\omega}\left(\mathbf{r}_{i}, \mathbf{r}_{j}\right)$. The normalized coherence function $C_{\omega}^{\prime}\left(\mathbf{r}_{1}, \mathbf{r}_{2}\right)$ is defined in this paper as ${ }^{18}$

$$
C_{\omega}^{\prime}\left(\mathbf{r}_{1}, \mathbf{r}_{2}\right)=\frac{C_{\omega}\left(\mathbf{r}_{1}, \mathbf{r}_{2}\right)}{\sqrt{C_{\omega}\left(\mathbf{r}_{1}, \mathbf{r}_{1}\right) C_{\omega}\left(\mathbf{r}_{2}, \mathbf{r}_{2}\right)}},
$$

and has the advantage of removing from the function the dependence on the noise level at the given frequency. For the aspects that are relevant to this study, the coherence function is discussed in further detail in the remainder of this paper; an extensive body of studies, including applications to marine ambient noise, is available in the literature. ${ }^{18-28}$

\section{FREQUENCY BASED COHERENCE-FUNCTION EXTENSION}

\section{A. Algorithm}

This section introduces an algorithm for extending the coherence function beyond the physical length of the array, which in the remainder of this paper will be called "frequency based extension" (FBE). The treatment begins by describing the algorithm, and then proceeds to justify its use by means of both theoretical and empirical arguments. In the following treatment, the symbol $C_{\omega}(z)$ introduces a more compact notation $C_{\omega}(z)=C_{\omega}\left(\mathbf{r}_{1}, \mathbf{r}_{2}\right)$, where it is assumed that $\mathbf{r}_{1}=(0,0)$ and $\mathbf{r}_{2}=(0, z)$-i.e., the hydrophone pair is assumed to be aligned with the $z$ axis, with the first hydrophone at $z=0$.

In FBE, the normalized coherence function $C_{\omega_{0}}^{\prime}\left(z_{1}\right)$ between two sensors is obtained, if an estimate of $C_{\omega_{1}}^{\prime}\left(z_{0}\right)-$ where $\omega_{1}>\omega_{0}$ and $z_{1}>z_{0}$-is available, with the condition

$$
\omega_{1} z_{0}=\omega_{0} z_{1}
$$

or, equivalently, $z_{0} / \lambda_{1}=z_{1} / \lambda_{0}$. More formally: For an $M$ element array, the maximum spacing for which the coherence function can be measured from data is the array length $z_{0}=(M-1) d$, and the (extended) value of the function at $z_{1}=n d$-where $n$ is an integer such that $n \geq M$ - can be obtained by assuming

$$
\begin{aligned}
& C_{\omega_{0}}^{\prime}(n d)=C_{\omega_{1}}^{\prime}[(M-1) d], \\
& \omega_{1}=n \omega_{0} /(M-1) .
\end{aligned}
$$

The remainder of this paper provides the theoretical and empirical basis of this algorithm, as well as examples of its application to both simulated and measured data.

\section{B. Modeling and application: Halfspace bottom}

It is easier to start discussing the FBE algorithm by considering the simple case of a bottom composed of a single material of constant acoustic properties, extending indefinitely in depth-in the remainder of this paper, this bottom type will be referred to as "halfspace." Furthermore, it is useful at this point to introduce an integral expression, originally derived by Harrison ${ }^{27,28}$ using a ray based approach, for the unnormalized noise vertical coherence. For two vertically separated hydrophones at spacing $z$, at a given frequency $\omega$ Harrison's equation can be written as a function of the sole angular variable $\theta:{ }^{11}$

$$
\begin{aligned}
C_{\omega}(z)= & \int_{0}^{\pi / 2} \frac{2 \pi\left(c_{r} / c_{s}\right) \sin \theta \cos \theta}{1-R_{s}(\theta) R(\theta) e^{-a s_{c}(\theta)}}\left\{e^{i(\omega / c) z \sin \theta} e^{-a s_{p}(\theta)}\right. \\
& \left.+R(\theta) e^{-i(\omega / c) z \sin \theta} e^{-a\left[s_{c}(\theta)-s_{p}(\theta)\right]}\right\} d \theta
\end{aligned}
$$

In Eq. (10), $s_{c}$ and $s_{p}$ are the complete (surface-bottom-surface) and partial (surface-sensor) ray-path lengths, whose dependence on $\theta$ is determined by the sound-speed profile in the water column; $R$ and $R_{S}$ are the bottom and surface power reflection coefficients, and $c_{r}$ and $c_{s}$ are the sound speed at the receiver and at the surface, respectively. In general, besides the ray angle, the reflection coefficients are also a function of frequency, but for the sake of simplicity this dependence will not be indicated explicitly. Note that $a$ is the power attenuation per unit length along the ray path, and the model assumes that the hydrophones are "close," so that a single ray path and sound speed can be defined for the sensor pair. 
Since the reflection coefficient of a halfspace bottom is independent of frequency, ${ }^{1}$ if one neglects the frequency dependence of $a$ (an acceptable assumption, as shown later), in this case the integrand of $C_{\omega}(z)$ does not depend on $\omega$ and $z$ separately, but rather on the term $\omega z / c=2 \pi z / \lambda$, where the familiar ratio of sensor spacing to wavelength appears. This is confirmed by the example provided in Figs. 2 and 3: Both plots show the normalized coherence function $C_{\omega}^{\prime}$ computed at selected frequency values using Eqs. (7) and (10), for a halfspace bottom (see case HS in Table I for the physical properties of the water column and the bottom). Each curve is plotted using 391 points over $0 \leq z \leq 5.85 \mathrm{~m}$, corresponding to an inter-sensor spacing of $0.015 \mathrm{~m}$. The markers show the positions of sensors number 10,20, and 40 in an array of spacing $d=0.15 \mathrm{~m}$ (this value is used in the simulations shown later in Sec. IV).

In Fig. 2, the curves are plotted as a function of sensor spacing $z$ and show the familiar decay along the horizontal axis, with more oscillations included over the array aperture as the frequency increases. However, the dependence of the coherence on the $z / \lambda$ ratio is better illustrated by the $C_{\omega}^{\prime}(z / \lambda)$ curves shown in Fig. 3, where, given the quantity on the horizontal axis, at a lower frequency two consecutive points of a curve are closer than they are at a higher frequency. The plots show that, aside from the slight amplitude differences due to the inclusion of volume attenuation in Eq. (10)—which is at the basis of the model used to generate these plots-both the real and the imaginary part of $C_{\omega}^{\prime}(z / \lambda)$ overlap almost perfectly, regardless of frequency. However, due to the difference in wavelength, the curves at higher frequencies extend farther to the right on the horizontal axis than those at lower frequencies. Using Fig. 3 for reference, understanding how the FBE algorithm works becomes straightforward, in this case: For example, the maximum spacing for a 10-element array is $z_{0}=1.35 \mathrm{~m}$, and an additional ("nonphysical") sensor

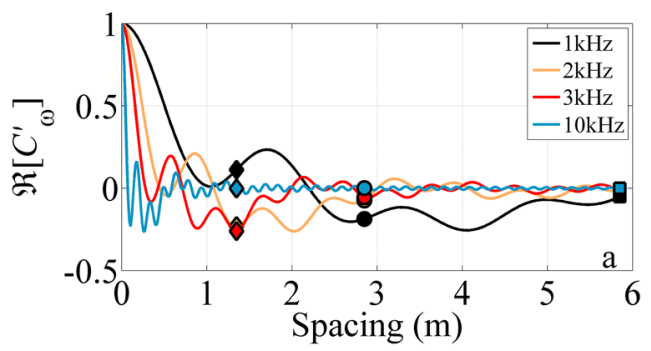

(a)

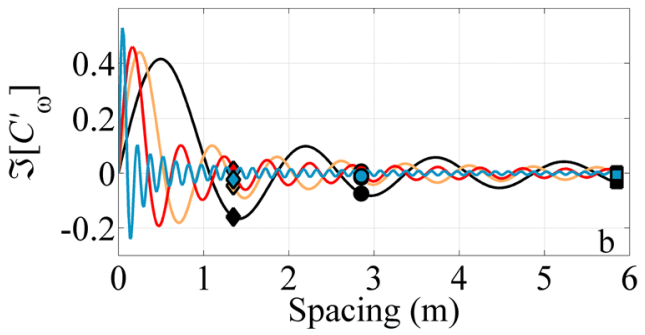

(b)

FIG. 2. (Color online) Halfspace bottom (case HS): Normalized coherencefunction real (a) and imaginary (b) part at several frequencies, as a function of sensor spacing $z$. The markers indicate the positions of sensors number 10 (diamond), 20 (circle), and 40 (square) for an array of spacing $d=0.15 \mathrm{~m}$.

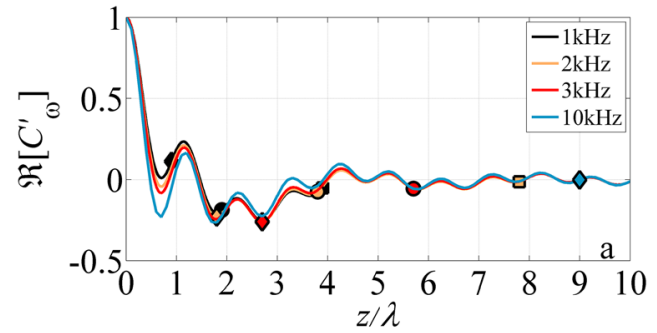

(a)

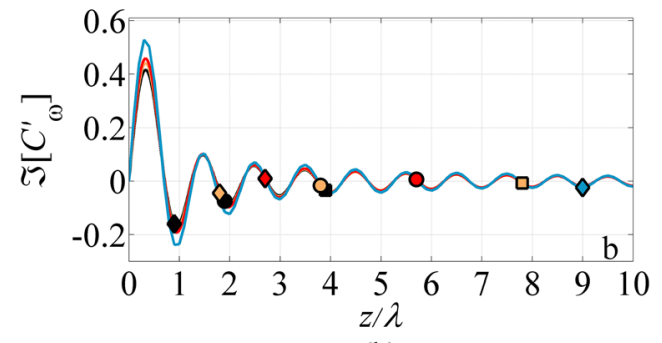

(b)

FIG. 3. (Color online) Halfspace bottom (case HS): Normalized coherencefunction real (a) and imaginary (b) part at the same frequencies as in Fig. 2, as a function of the $z / \lambda$ ratio. The markers indicate the same sensors as in Fig. 2, but given the quantity on the horizontal axis, at a lower frequency two consecutive points of a curve are now closer than they are at a higher frequency, and the curve corresponding to a higher frequency reaches higher values on the horizontal axis. In each plot, the curves for different frequencies overlap very closely.

number 11 would be at $z_{1}=1.50 \mathrm{~m}$ from sensor number 1 . If one assumes $f_{0}=\omega_{0} / 2 \pi=1 \mathrm{kHz}$, then $z_{1} / \lambda_{0}=1$, and Eq. (8) yields $f_{1}=(1.50 / 1.35) f_{0}=1.111 \mathrm{kHz}$. The point corresponding to $z / \lambda=1$ on the $1.111 \mathrm{kHz}$ curve is then used to estimate the coherence at the position of the "nonphysical" sensor number 11 on the $1 \mathrm{kHz}$ curve. The maximum available value for $z_{0}$ is chosen on purpose in this example, so as to minimize the difference between $\omega_{0}$ and $\omega_{1}$, ensuring that the error between the two $C_{\omega}^{\prime}(z / \lambda)$ curves is minimized.

\section{Considerations on layered bottoms}

Although the treatment above relies on the fact that the bottom reflection coefficient is independent of frequency, use of the FBE algorithm prior to BL estimation (treated extensively in Secs. IV and V below) improves the quality of the results also in the case of layered bottoms, where the frequency dependence of the reflection coefficient can be dramatic. The reason for this is not immediately apparent from theoretical

TABLE I. Water-column and bottom configuration for the simulated cases; $\Delta$ is the layer thickness, $\rho$ is the density, $\alpha_{c}$ is the compressional volume attenuation, and $\lambda$ is the wavelength.

\begin{tabular}{lcccc}
\hline \hline & $\Delta(\mathrm{m})$ & $c_{p}(\mathrm{~m} / \mathrm{s})$ & $\rho\left(\mathrm{kg} / \mathrm{m}^{3}\right)$ & $\alpha_{c}(\mathrm{~dB} / \lambda)$ \\
\hline Water & 170 & 1500 & 1000 & $1 \times 10^{-4}$ \\
HS & $\infty$ & 1565 & 1500 & 0.2 \\
1L-Layer & 0.5 & 1650 & 1500 & 0.2 \\
1L-Halfspace & $\infty$ & 1700 & 2000 & 0.5 \\
2L-Layer \#1 & 0.5 & 1565 & 1500 & 0.2 \\
2L-Layer \#2 & 3 & 1625 & 1700 & 0.3 \\
2L-Halfspace & $\infty$ & 1800 & 2000 & 0.5 \\
\hline \hline
\end{tabular}


models, which present $C_{\omega}(z)$ either in integral form, such as Eq. (10), or as a series expansion. ${ }^{23}$ The expression of $C_{\omega}(z)$ as the combination of a direct and an inverse Fourier transform between the hydrophone spacing $z$ and the vertical wavenumber (and therefore, the frequency $\omega$ ) domains ${ }^{11}$ makes the connection between the two quantities explicit, but this fact alone does not fully explain why FBE is so effective in aiding BL estimation. An approach based on both theoretical and empirical considerations is proposed in this study.

As a starting point, the $C_{\omega}^{\prime}(z / \lambda)$ curves obtained from Eq. (7) and Eq. (10) for the layered bottom types $1 \mathrm{~L}$ and $2 \mathrm{~L}$ in Table $I$ are shown in Figs. 4 and 5. One of the most remarkable features in the plots is that, while the real parts of $C_{\omega}^{\prime}(z / \lambda)$ appear to vary significantly between the two cases, the imaginary parts have a much more "regular" behavior, and appear to differ mostly in the amplitude of their oscillations. This can be explained starting from Eq. (10), and introducing the simplifying hypotheses of an isospeed water column $\left(c_{r}=c_{s}=c\right)$ with negligible volume attenuation $(a \approx 0)$ and a unit-value surface reflection coefficient $\left(R_{s} \approx 1\right)$, which yields the simplified expression

$$
C_{\omega}(z)=\int_{0}^{\pi / 2} \frac{\pi \sin 2 \theta}{1-R(\theta)}\left[e^{i(\omega z / c) \sin \theta}+R(\theta) e^{-i(\omega z / c) \sin \theta}\right] d \theta .
$$

Now, by expressing the exponentials in trigonometric form, one can separate the real and imaginary parts of the integral:

$$
\begin{aligned}
& \operatorname{Re}\left[C_{\omega}(z)\right]=\int_{0}^{\pi / 2} \pi \sin 2 \theta \frac{1+R}{1-R} \cos \left(\frac{\omega z}{c} \sin \theta\right) d \theta \\
& \operatorname{Im}\left[C_{\omega}(z)\right]=\int_{0}^{\pi / 2} \pi \sin 2 \theta \sin \left(\frac{\omega z}{c} \sin \theta\right) d \theta .
\end{aligned}
$$

The most apparent feature in Eq. (12) is the absence of the reflection coefficient in $\operatorname{Im}\left[C_{\omega}^{\prime}(z / \lambda)\right]:$ In this simplified

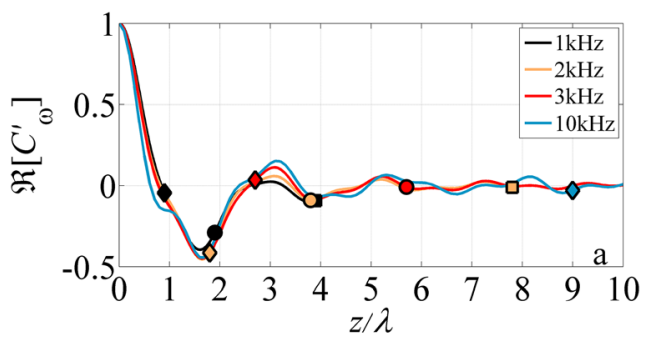

(a)

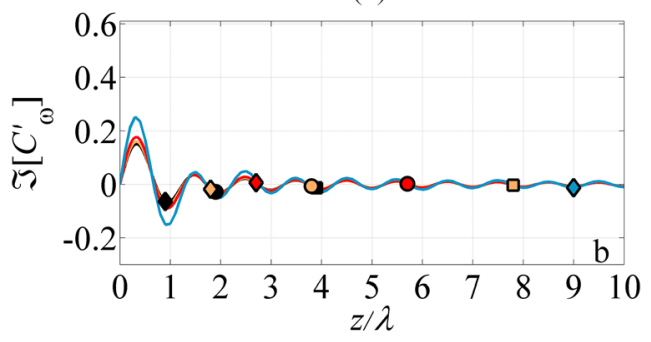

(b)

FIG. 4. (Color online) Single layer over halfspace (case 1L): Normalized coherence-function real (a) and imaginary (b) part at several frequencies, as a function of the $z / \lambda$ ratio. The markers are positioned as in Fig. 3. The curves in panel $b$ differ from their counterparts in Figs. 3 and 5 mainly in amplitude.

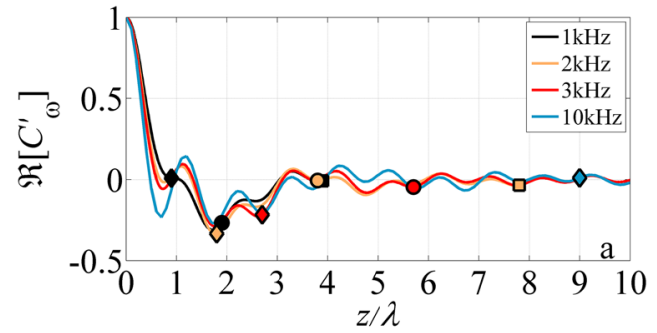

(a)

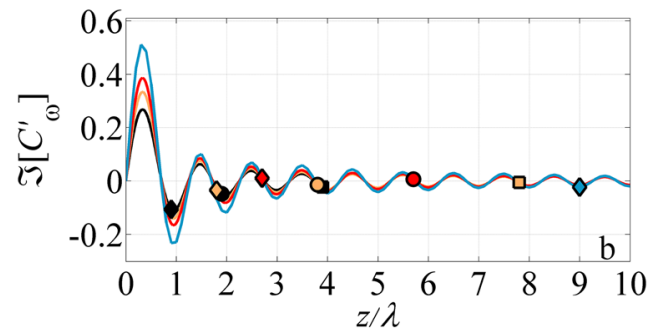

(b)

FIG. 5. (Color online) Double layer over halfspace (case 2L): Normalized coherence-function real (a) and imaginary (b) part at several frequency values, as a function of the $z / \lambda$ ratio. The markers are positioned as in Fig. 3. The curves in panel (b) differ from their counterparts in Figs. 3 and 4 mainly in the amplitude.

model, the imaginary part of $C_{\omega}^{\prime}(z / \lambda)$ is independent of the bottom reflection properties, and its dependence on $\omega$ is only present as the $\omega z$ product. This conclusion is confirmed by Figs. 4 and 5, where the behavior of the imaginary part curves is analogous to that observed in the halfspace case (see Fig. 3).

Furthermore, Eq. (12) indicates that the differences due to the bottom type should manifest themselves in the real part curves, which, in fact, show clear differences between the two cases. However, it should be noted that, even for these layered bottoms, if one considers two $\operatorname{Re}\left[C_{\omega}^{\prime}(z / \lambda)\right]$ curves corresponding to "close" frequency values, the points corresponding to the same $z / \lambda$ value on the two curves will be close too. In other words, although the curves can be proven theoretically to overlap perfectly (except for the frequency dependence of $a$ ) only for a halfspace bottom, they still appear to vary smoothly with frequency, in the case of layered bottoms. The results presented in Secs. IV and V below confirm that this reasonable hypothesis holds, and that the FBE algorithm does help improve the BL estimates from short arrays.

\section{APPLICATION TO BOTTOM-LOSS ESTIMATION: SIMULATION}

Simulation can be useful at this point to investigate further the dependence of the coherence function on signal and array physical parameters. In the remainder of this paper, simulated data have been produced using OASN, the surfacenoise module of the OASES ${ }^{29}$ package. By implementing a numerical solution to the full wave equation for range independent, stratified media - as opposed to implementing an analytical model-OASES produces directly the $\hat{\mathbf{C}}_{\omega}$ matrix of Eq. (3), providing a more realistic approximation to what an estimate of the coherence function from measured data 
would be. For its simulation part, this study presents the application of OASN to the three different types of bottom already investigated above: Halfspace (identified by "HS"), single layer over halfspace ("1L"), and two layers over halfspace ("2L"). The bottom properties for each case are shown in Table I.

\section{A. Considerations on array configuration and the bandwidth of the estimated bottom loss}

Arrays suitable for deployment on AUVs of the class considered in this study should reasonably have a length no larger than $2 \mathrm{~m}$, but the applicability of Harrison and Simons' technique to such arrays, especially at frequencies below $10 \mathrm{kHz}$ has been shown to be problematic, due to the severe deterioration of the beamformer's angular resolution. ${ }^{10}$ High resolution bottom-loss estimation (HR-BL) has recently shown that $\mathrm{BL}$ estimates from Harrison and Simons' technique can be improved, by replacing the CBF with a more sophisticated technique, which exploits the physical properties of the surface generated noise field. ${ }^{11}$ However, the challenge posed by BL estimation with AUV deployable arrays in the $500-5000 \mathrm{~Hz}$ frequency range makes any further performance improvement highly desirable.

As an example of an effective application of FBE, this section shows how it can improve significantly the performance of short arrays in passive bottom-loss estimation. The main advantage is the possibility of improving the grazingangle resolution of the estimated bottom loss to a level that the original technique could only achieve by means of a longer array. A subtler advantage is a more efficient use of the bandwidth of current acquisition systems, explained below.

The beamforming operation imposes a practical limitation on BL estimation: The upper limit on the frequency range over which data can be used (hereafter referred to as "array design frequency"). For conventional beamforming on a line array, this limit is determined by the inter-sensor spacing: The maximum frequency at which the array can operate as a directional antenna corresponds to a wavelength that is twice the spacing. For instance, assuming a sound speed in water of $1500 \mathrm{~m} / \mathrm{s}$ an array whose sensors are spaced $0.15 \mathrm{~m}$ has a design frequency of $5 \mathrm{kHz}$. The appearance of grating lobes in the beam pattern makes it impossible to estimate the bottom loss above the array design frequency.

However, with the sampling rates afforded by current acquisition systems, the array design frequency usually falls well below the Nyquist frequency, leaving a sizeable fraction of the data unused, for the purpose of bottom-loss estimation. In FBE, data from higher frequencies are used to estimate the noise spatial coherence function at a lower frequency, for values of the sensor spacing beyond the physical length of the array. As Eq. (9) shows, if one wants to double the number of sensors, and therefore double the array length, and estimate the BL up to the array design frequency $f_{d}=c / 2 d$, it is necessary to have data available from the physical sensors up to a frequency that is roughly $2 f_{d}$. By doing so, the angular resolution of the bottom-loss estimate can be improved, often making use of data at frequencies otherwise not utilized for beamforming.

\section{B. Application to simulated data}

In this section, the application of FBE to passive BL estimation is investigated through simulation. This ensures the a priori knowledge of the bottom and the water column (a luxury that experiments on the field usually cannot afford), making it possible to compare the results to modelbased predictions. Since the goal is BL estimation, in this study the reference is provided by a model, presented by Jensen et al., ${ }^{1}$ that predicts the power reflection coefficient of a horizontally stratified fluid bottom of known physical properties, as a function of frequency and grazing angle.

For each of the cases introduced above, the model of Jensen et al. has been run to provide the predicted BL to be used as reference. The CSD matrices produced by OASN for the same bottom types have been processed to estimate the BL via CBF, as in Harrison and Simons' original technique, using a 24-element array with $0.15 \mathrm{~m}$ spacing. The procedure has been repeated using only the first 12 elements of the array, to show how the estimated BL is impacted by a significant reduction of the array length. Finally, the same $12 \times 12$ CSD matrices have been used to estimate the 12-point coherence function by diagonal averaging, ${ }^{11}$ and this has been extended as described in Sec. III up to the length of the original array, i.e., adding 12 points corresponding to "synthetic" sensors beyond the length covered by the "physical" sensors. In the examples presented in this section, the frequency domain has been sampled with 680 bins of $50 \mathrm{~Hz}$ width between $50 \mathrm{~Hz}$ and $34 \mathrm{kHz}$. In general, the frequency value required to apply Eq. (9) will not fall at the center of one of the chosen frequency bins. The results presented in the remainder of this paper have been obtained by simple linear interpolation of the coherence function between the closest available frequency bins. The HR-BL algorithm has then been applied to the extended coherence function to estimate the BL.

The results obtained by the procedure outlined above for case HS are shown in Fig. 6. Panels (a) and (c) show the known limitations of beamforming: While the predicted BL is perfectly frequency independent, the 24-element beamformer-corresponding to a $3.45 \mathrm{~m}$ aperture-places the critical angle correctly only at the higher frequencies. With decreasing frequency, the beams become wider, and the decreased angular resolution causes an area of substantial BL underestimation, which extends to cover the entire grazing angle range at the bottom of the plot. The design frequency for this array is $5 \mathrm{kHz}$, and at this frequency, around normal incidence, the BL estimate drops to zero, due to the grating lobes that appear in the beam pattern. When the aperture is reduced to $1.65 \mathrm{~m}$ [12 elements, see panel (b)], all the limitations described above are magnified. Finally, panel (d) in Fig. 6 shows the BL estimated by HR-BL with data from the same 12 sensors, but after extending the coherence function by FBE back to 24 elements: The plot shows a virtually complete recovery of the information lost by the shorter array. 

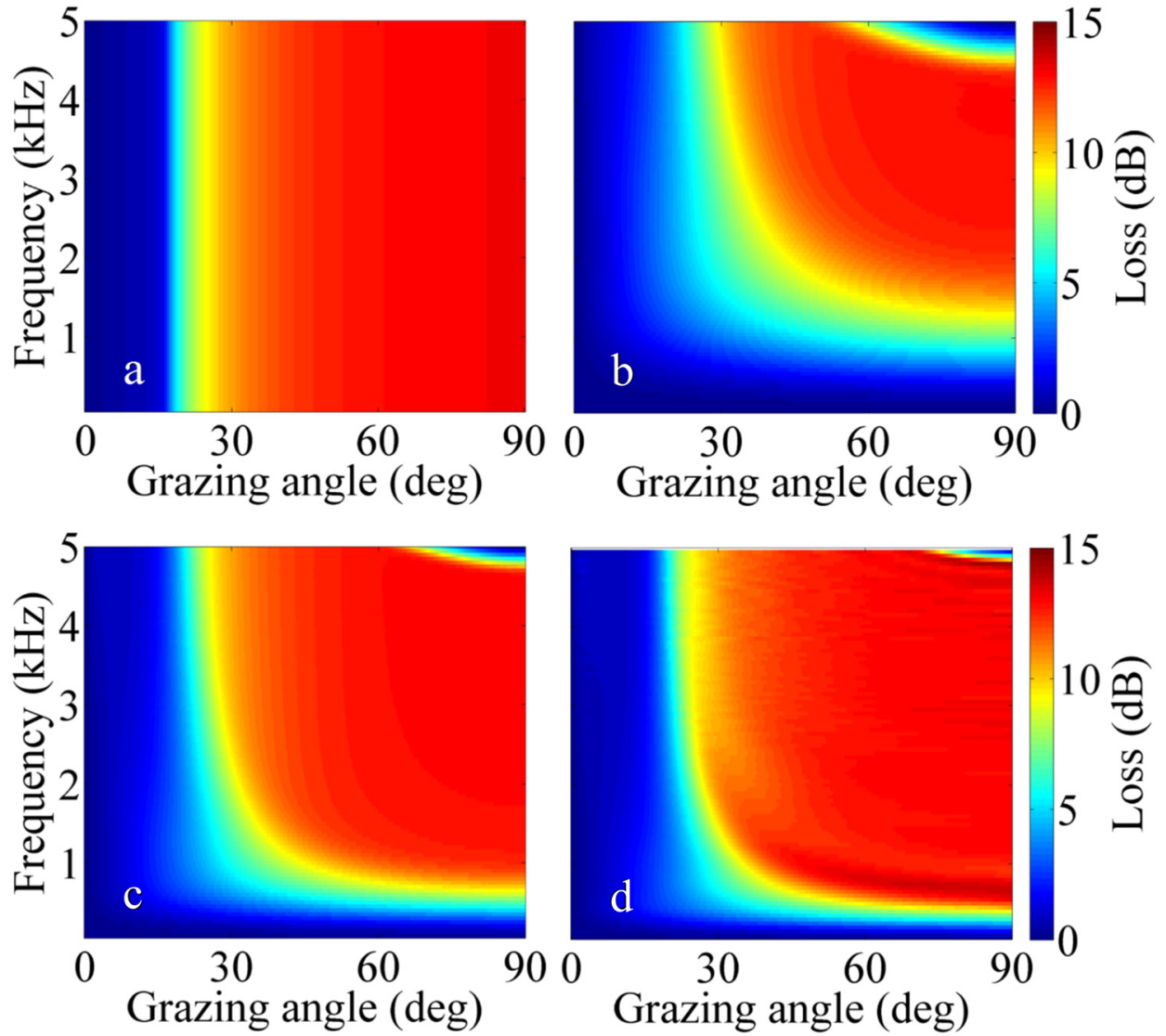

FIG. 6. (Color online) Halfspace bottom (case HS): BL predicted using the reflection coefficient given by the model of Jensen et al. (a); BL estimated from OASN data using CBF over 12 (b) and 24 (c) sensors, and BL estimated by HR-BL over 12 sensors extended to 24 by FBE (d).
Given the analysis presented in Sec. III, it is not surprising to see that FBE performs well on a halfspace bottom. For a layered bottom, one could expect the more pronounced dissimilarity of the coherence-function real part to make the application of FBE more difficult. This has been preliminary investigated through a number of simulations, of which the results for cases 1L and 2L, shown in Figs. 7-9, respectively, are presented as a sample. These results have been produced following the same procedure as the one described above for case HS.

For layered bottoms, the predicted BL presents more "structure" than for a halfspace. Such structure can be rather fine, as shown, for instance, in case 1L [see Fig. 7(a)] at the critical angle (the angle above which the BL becomes significant), and, in case 2L (see Fig. 9), in the thin striations overlapping with the three wide striations. Although the BL estimated by Harrison and Simons' technique from simulated data are very "clean," compared to what is usually observed when the technique is applied to experimental data, such fine features fall beyond the resolution of any of the processing techniques presented in this paper. In general, the BL estimated in the reference case [24-element CBF, panel (c) in Figs. 7 and 9] appears to be a "smeared" version of the modelled BL, and presents the area of significant BL underestimation at the lower frequencies described above. These effects are expected, as they are due to the finite angular resolution of the beamformer, and are accentuated when moving to the half-length array [12 elements, panel (b) in Figs. 7 and 9]. The BL estimated by HR-BL using the data from the same 12 elements-after extending the estimated coherence function at the locations of 12 additional "synthetic" sensors - shows in both layered-bottom cases [panel (d) in Figs. 7 and 9] a significant recovery of the information lost by the 12-element CBF. For comparison, Fig. 8 shows the BL estimated for case L1 by HR-BL alone. Using the 12element array, HR-BL alone achieves a significant improvement over CBF [compare Figs. 7(b) and 8(a)]. However, it remains far from the performance it achieves after FBE extension from 12 to 24 elements [see Fig. 7(d)], which in turn is very close to the BL estimated by HR-BL alone using the full, 24-element array [shown in Fig. 8(b)].

\section{APPLICATION TO MEASURED DATA}

This section presents the results of applying FBE to passive BL estimation from actual data measured in three different experiments at sea by the NATO-STO Centre for Maritime Research and Experimentation. The data refer to three different sites and arrays, and the data set identifiers used in the remainder of this paper, as well as the basic characteristics of each data set and array, are listed in Table II. The medium frequency array (MFA) and VLA data are from the experimental campaign named Boundary 2003, while the slim vertical array (SLIVA) data set was recorded during the REP14-MED experiment of 2014.

The emphasis in this study is in showing how FBE can improve the performance of a short array in BL estimation. For this reason, rather than comparing the results to a ground truth that in the case of the measured data is rather uncertain, the comparison is carried out between the full 32-element array, a subarray including only a subset of the original elements, and the same subarray extended to the original length 

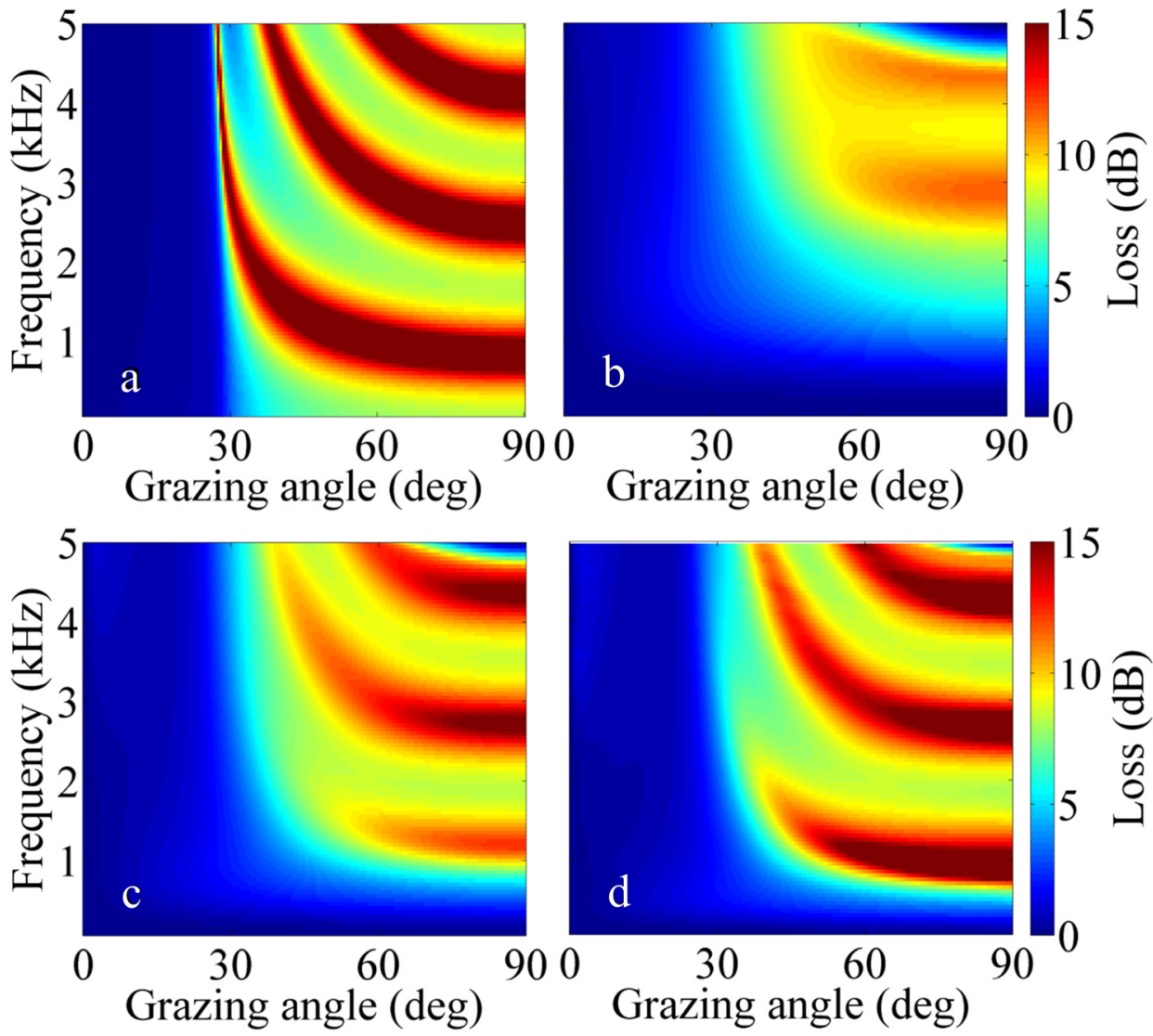

FIG. 7. (Color online) Single layer over halfspace (case 1L): BL predicted using the reflection coefficient given by the model of Jensen et al. (a); BL estimated from OASN data using CBF over 12 (b) and 24 (c) sensors, and BL estimated by HR-BL over 12 sensors extended to 24 by FBE (d). The very fine structure visible in the modelled $\mathrm{BL}$ around the critical angle falls beyond the resolution of any of these techniques. by using FBE to estimate the coherence function at the location of the missing original sensors.

All the CSD matrices used in this study correspond to 5min data averages. The plots in Figs. 10 and 11 compare the $\mathrm{BL}$ estimated by applying different techniques to data recorded over the full 32-element arrays of the Boundary experiment, and to a 20-element subarray. In these examples, the magnitude of the extension (from 20 to 32 elements) used by the FBE algorithm is limited by the low Nyquist frequency, which in particular, prevents the recovery of the BL estimate up to the design frequency $(4166 \mathrm{~Hz}$ assuming a sound speed of $1500 \mathrm{~m} / \mathrm{s}$ ) for the MFA data set. In all cases the deterioration due to the reduced array length is visible in the same terms as already described above for the synthetic data, and FBE coupled with HR-BL proves to be able to recover in the $\mathrm{BL}$ most of the information lost by applying the CBF to the short array, as in Harrison and Simons' original technique. In the MFA data, some high loss striations appear at very low grazing angles in the FBE result in Fig. 10. It is unclear whether these represent an actual feature of the bottom, but analysis of the other panels in the figure, shows that these features are present in the BL estimated by the other techniques, and are simply emphasized by the higher resolution of the HR-BL algorithm.

Finally, the VLA results in Fig. 11 may not appear to be as "dramatic" as the MFA ones; this is due to the nature of the data. The analysis of these data shows that the surface noise field is contaminated by other contributions, a circumstance to which the HR-BL algorithm is known to be sensitive. ${ }^{11}$ To alleviate the consequences of this, a Hanning taper
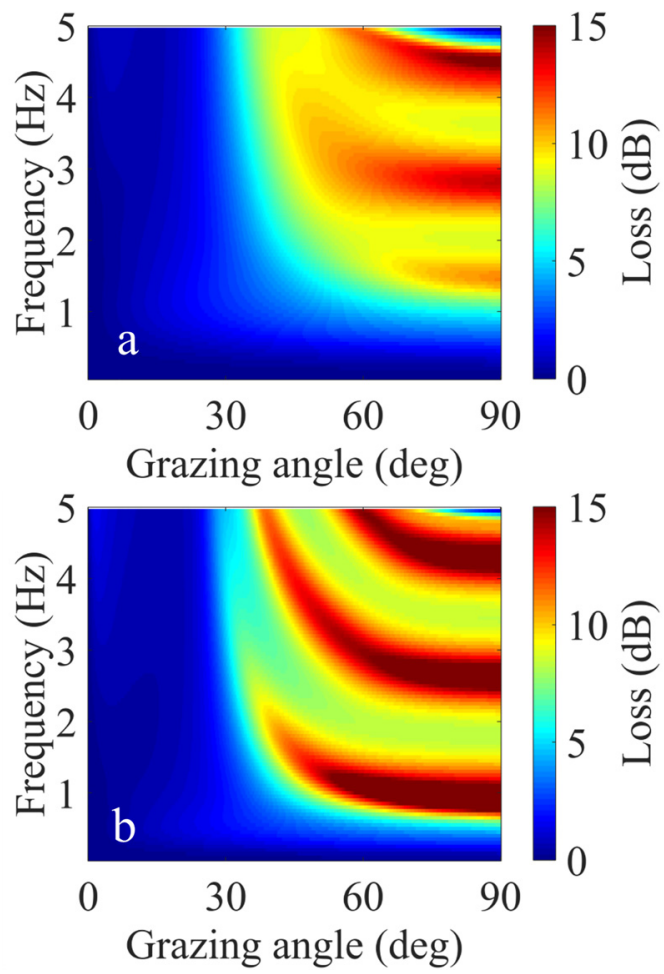

FIG. 8. (Color online) Single layer over halfspace (case 1L): BL estimated from OASN data using HR-BL over 12 (a) and 24 (b) sensors. Using 12 sensors, HR-BL shows a clear improvement over CBF [compare panel (a) here to Fig. 7(b)]. However, the performance of the algorithm remains well below the result shown in Fig. 7(d), obtained after extending the array to 24 elements by FBE, which is very close to that in panel (b) here. 

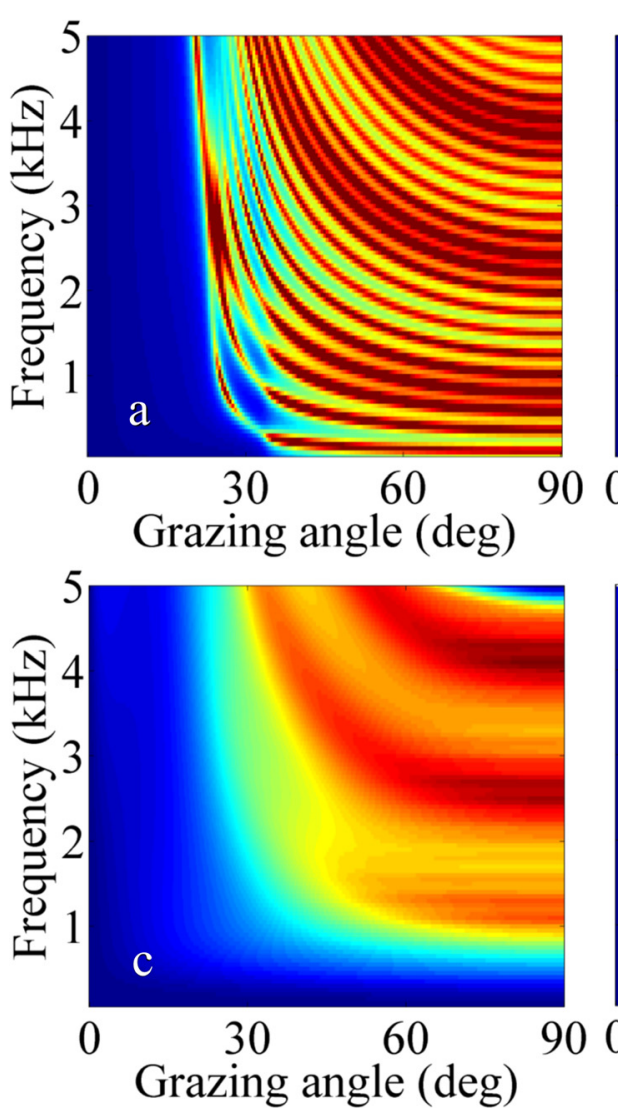

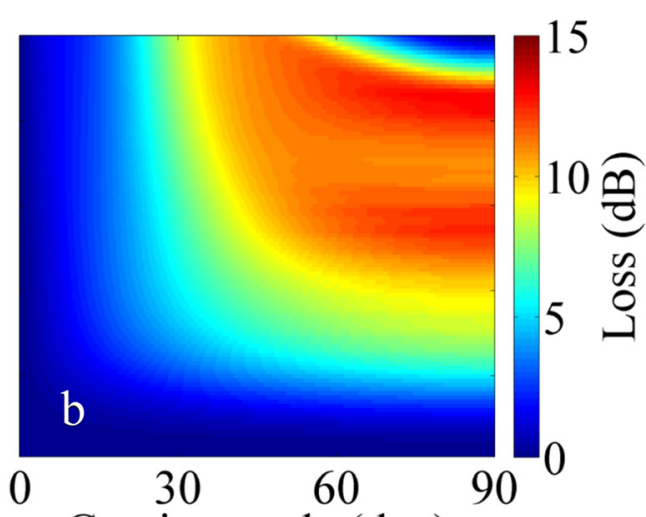

Grazing angle (deg)

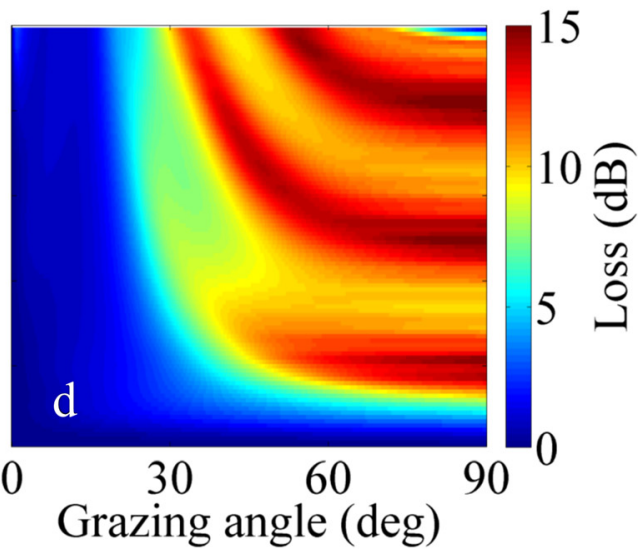

FIG. 9. (Color online) Double layer over halfspace (case $2 \mathrm{~L}$ ): BL predicted using the reflection coefficient given by the model of Jensen et al. (a). BL estimated from OASN data using $\mathrm{CBF}$ over 12 (b), and 24 (c) sensors, and BL estimated by HR-BL over 12 sensors extended to 24 by FBE (d). Due to the limited resolution afforded by the array, the very fine striations overlapping the three main striations are just barely visible in panels (c) and (d). was applied to the CSD matrix prior to HR-BL processing, a procedure that limits the performance of the algorithm. However, it should also be noted that although this low frequency array, with an inter sensor spacing of $0.5 \mathrm{~m}$, can be more challenging for FBE, it is also an unlikely candidate for small AUV deployment.

Data-bandwidth limitation is a less important problem today, with data acquisition systems that are capable of much
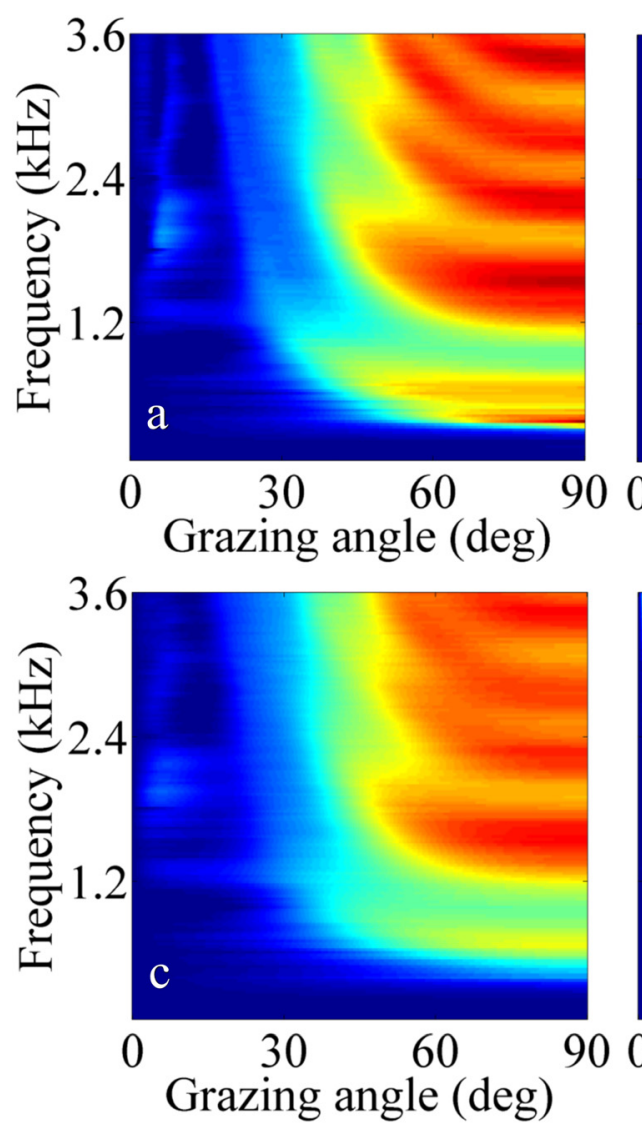
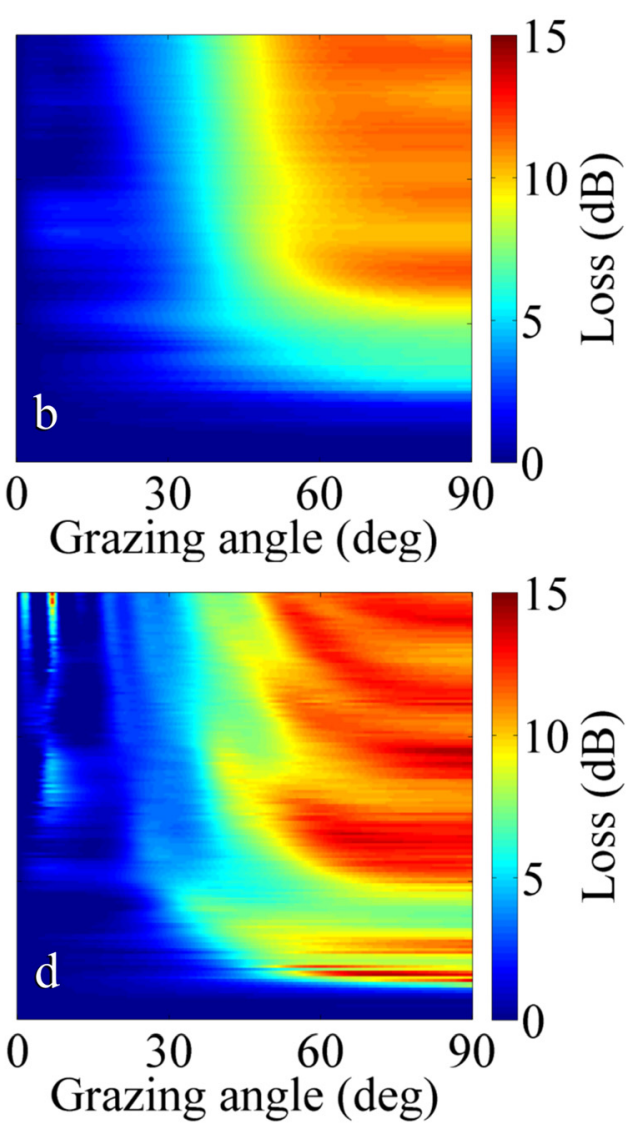

FIG. 10. (Color online) Boundary 2003 MFA data: BL estimated by HRBL over 32 elements (a), by CBF over 20 (b) and 32 elements (c), and by HR$\mathrm{BL}$ after extending the coherence function estimated from 20 sensors to 32 sensors by FBE (d). The origin of the artifacts visible in panel (d) at low grazing angles is at present unclear. Although such artifacts appear to be emphasized by the combination of FBE and HR-BL, they are present also in the other panels, indicating that they are not a product of this particular technique. 
TABLE II. Data sets and array basic features.

\begin{tabular}{lccccc}
\hline \hline $\begin{array}{l}\text { Data } \\
\text { set ID }\end{array}$ & $\begin{array}{c}\text { No. of } \\
\text { elements }\end{array}$ & $\begin{array}{c}\text { Spacing } \\
(\mathrm{m})\end{array}$ & $\begin{array}{c}\text { Sampling } \\
\text { freq. (Hz) }\end{array}$ & $\begin{array}{c}\text { Design freq. } \\
(\mathrm{Hz}) \text { at } \\
\mathrm{c}=1500 \mathrm{~m} / \mathrm{s}\end{array}$ & $\begin{array}{c}\text { Deployment } \\
\text { type }\end{array}$ \\
\hline VLA-03 & 32 & 0.50 & 6000 & 1500 & Drifting \\
MFA-03 & 32 & 0.18 & 12000 & 4166 & Drifting \\
SLIVA-14 & 32 & 0.18 & 50000 & 4166 & Moored \\
\hline \hline
\end{tabular}

higher sampling rates. For instance, Table II shows that the SLIVA array has the same spacing as the MFA array, but a much higher sampling rate. In Fig. 12 the BL estimated using the full SLIVA array is compared to that obtained from an eight-element subarray which, at $1.26 \mathrm{~m}$ of length, would be a good candidate for deployment on small AUVs. The BL estimated using CBF on the subarray [see Fig. 12(b)] shows a significant loss of information, but in this case the sampling rate is high enough to "extend" the subarray back to 32 elements, therefore quadrupling the array length, and up to the array design frequency. Even in this rather extreme attempt, FBE appears to afford a significant recovery of information in the BL estimate [see Fig. 12(d)].

To conclude the experimental part of this study, it is important to stress that the quality of these results depends on the measured acoustic field being free of sources other than wind and wave noise. Furthermore, it should be noted that there can be features in the coherence function that do not manifest themselves in measurement if the array does not have an adequate length. In such cases, FBE may not recover such features, which may correspond to some details in the BL plot, but it will still provide an approximation to the general shape of the function, and its decay with increasing $z / \lambda$.

\section{CONCLUSIONS}

The analysis of an existing model of the spatial coherence function (as measured by a vertical line array in a natural surface-noise field) shows that the dependence of this function on the bottom reflection properties is mostly contained in the real part. Both the real and the imaginary part of the function depend strongly on the ratio of the sensorpair spacing to signal wavelength $(z / \lambda)$. However, due to the influence of the bottom reflection properties, the real part shows a stronger dependence on bottom type and, for the same bottom, on frequency.

Based on these considerations, in the case of a frequency-independent bottom reflection coefficient (such as that of a halfspace bottom), a simple technique can be envisioned to extend the coherence function to values of the sensor spacing that are beyond the physical length of the array, by making use of data at higher frequencies, and provided that the spacing-to-wavelength ratio is preserved. While some amount of error is expected when the technique is applied to a layered bottom, results show that, for the particular task of passive bottom-loss estimation, these errors are well within the margins of Harrison and Simons' technique. This appears to be due to the fact that the coherence function varies smoothly with frequency. Processing of both simulated and measured data by FBE coupled with HR-BL shows
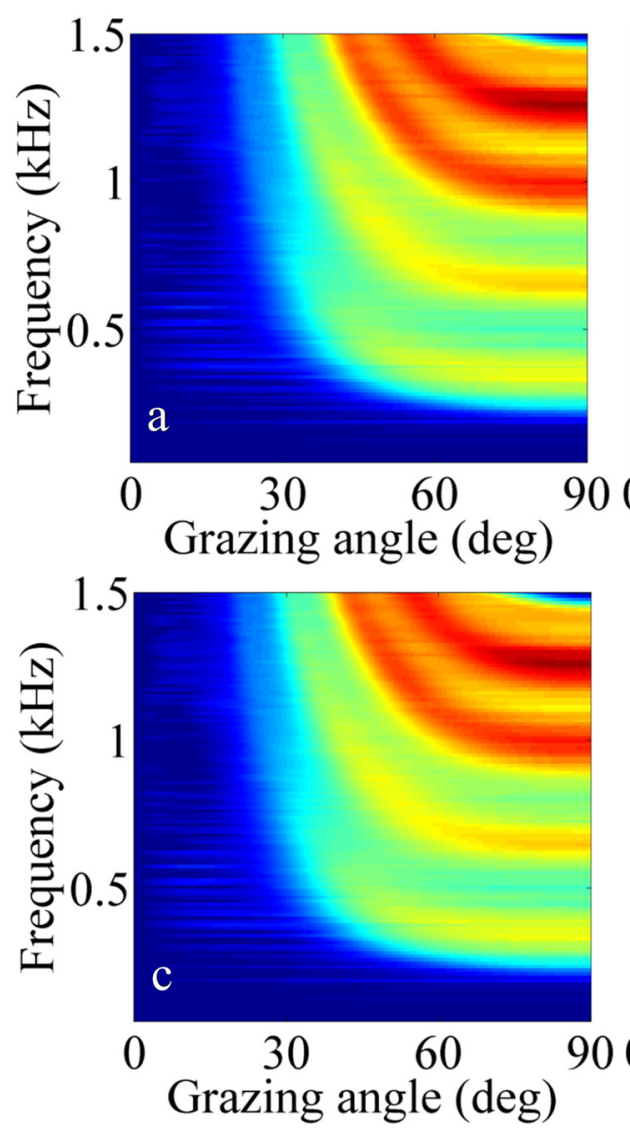

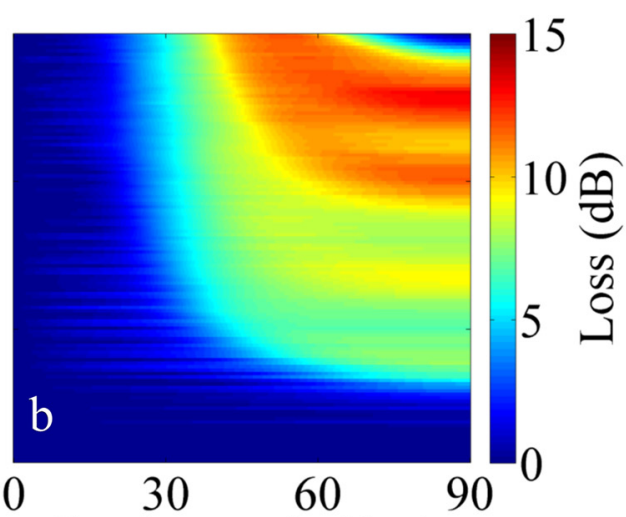

Grazing angle (deg)

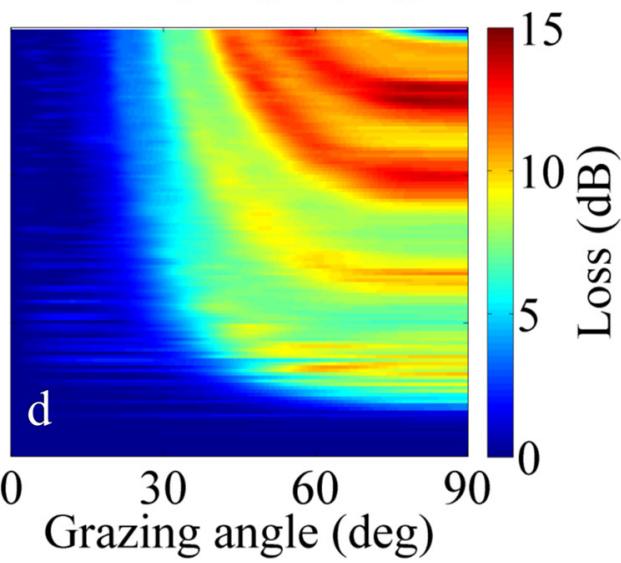

FIG. 11. (Color online) Boundary 2003 VLA data: BL estimated by HRBL over 32 elements (a), by CBF over 20 (b) and 32 elements (c), and by HRBL after extending the coherence function estimated from 20 sensors to 32 sensors by FBE (d). Due to contamination of the natural noise field by interferers (a condition to which HR-BL is known to be sensitive), a Hanning taper was applied to the CSD matrix before passing it to the HR-BL algorithm. 

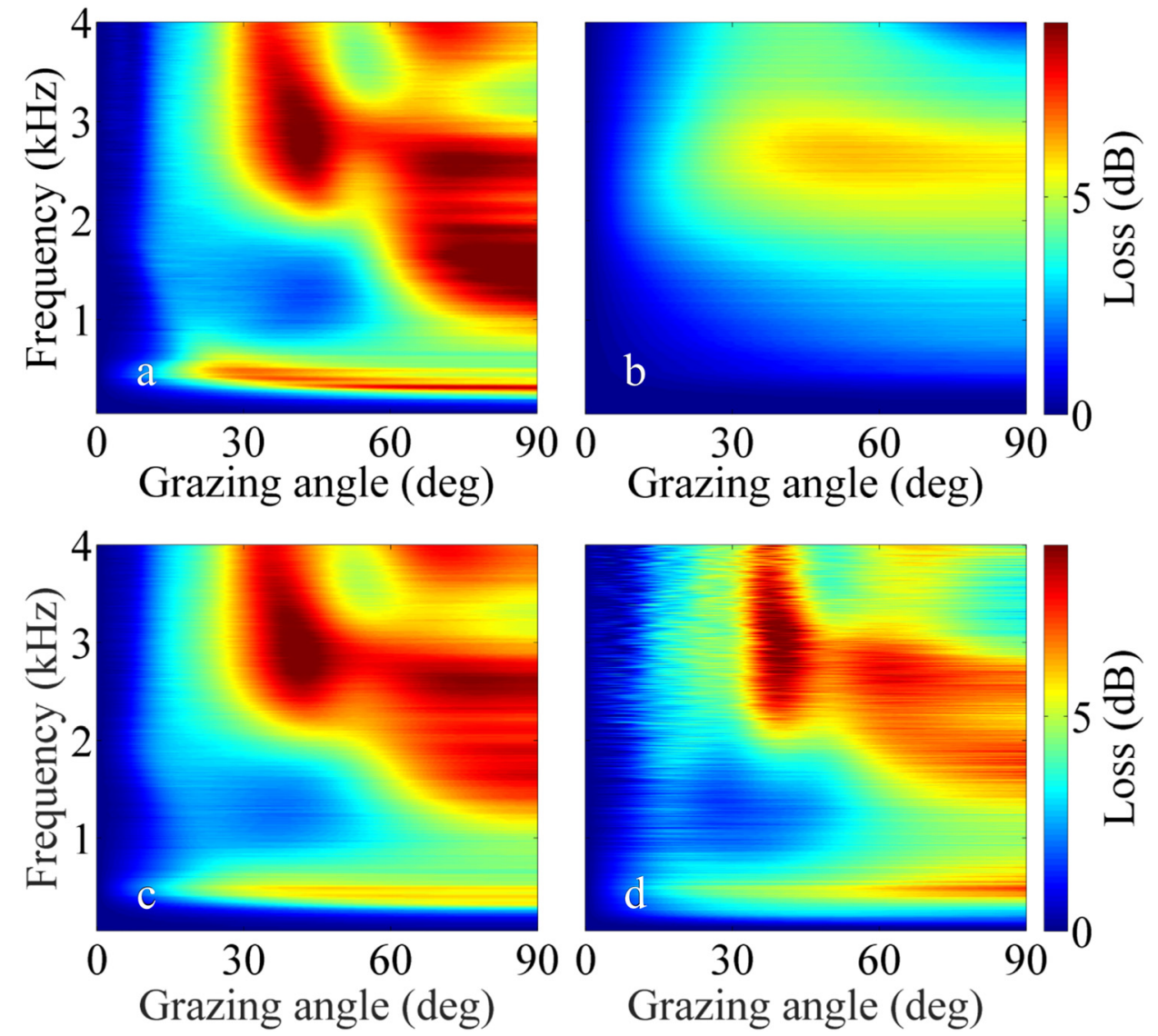

FIG. 12. (Color online) SLIVA data: BL estimated by HR-BL over 32 elements (a), by CBF over eight (b) and 32 elements (c), and by HR-BL after extending the coherence function estimated from eight sensors to 32 sensors by FBE (d). Despite the fact that the length of the array is being increased by a factor of 4 , the high sampling frequency affords the recovery of BL up to the array design frequency. that the information lost by a short array can be at least partially recovered, while making a more efficient use of the large bandwidth afforded by modern acquisition systems. An important prerequisite for the application of the approach illustrated in this paper is that the data be free of interference from sources other than wind and wave noise.

\section{ACKNOWLEDGMENTS}

The authors gratefully acknowledge the support of the Office of Naval Research Ocean Acoustics Program (ONROA Code 3211), and the Visiting Researcher Program of the NATO-STO Centre for Maritime Research and Experimentation (CMRE, formerly NATO Undersea Research Centre), which supported L.M. for part of this study. The authors also gratefully thank Reiner Onken, Scientist in Charge during the REP14-MED sea trial.

${ }^{1}$ F. B. Jensen, W. A. Kuperman, M. B. Porter, and H. Schmidt, "Fundamentals of ocean acoustics," in Computational Ocean Acoustics (Modern Acoustics and Signal Processing), 2nd ed. (Springer, New York, 2011), Chap. 1, pp. 38-50.

${ }^{2}$ R. Hamson, "The modelling of ambient noise due to shipping and wind sources in complex environments," Appl. Acoust. 51, 251-287 (1997).

${ }^{3}$ E. L. Hamilton, "Geoacoustic modeling of the sea floor," J. Acoust. Soc. Am. 68, 1313-1340 (1980).

${ }^{4}$ E. L. Hamilton and R. T. Bachman, "Sound velocity and related properties of marine sediments," J. Acoust. Soc. Am. 72, 1891-1904 (1982).

${ }^{5}$ C. Ferla and F. B. Jensen, "Are current environmental databases adequate for sonar predictions in shallow water?," in Impact of Littoral Environmental Variability on Acoustic Predictions and Sonar Performance, edited by N. G. Pace and F. B. Jensen (Springer Science+Business Media, Dordrecht, the Netherlands, 2002), pp. 555-562.
${ }^{6}$ S. E. Dosso, M. L. Yeremy, J. M. Ozard, and N. R. Chapman, "Estimation of ocean-bottom properties by matched-field inversion of acoustic field data," IEEE J. Ocean. Eng. 18, 232-239 (1993).

${ }^{7}$ A. Caiti, S. M. Jesus, and A. Kristensen, "Geoacoustic seafloor exploration with a towed array in a shallow water area of the Strait of Sicily," IEEE J. Ocean. Eng. 21, 355-366 (1996).

${ }^{8}$ M. Siderius, P. L. Nielsen, and P. Gerstoft, "Range-dependent seabed characterization by inversion of acoustic data from a towed receiver array," J. Acoust. Soc. Am. 112, 1523-1535 (2002).

${ }^{9}$ C. H. Harrison and D. G. Simons, "Geoacoustic inversion of ambient noise: A simple method," J. Acoust. Soc. Am. 112, 1377-1389 (2002).

${ }^{10} \mathrm{M}$. Siderius and C. Harrison, "High-frequency geoacoustic inversion of ambient noise data using short arrays," in High Frequency Ocean Acoustics Conference, edited by M. B. Porter, M. Siderius, and W. A. Kuperman, AIP Conf. Proc. 728, 22-31 (2004).

${ }^{11}$ L. Muzi, M. Siderius, J. Quijano, and S. Dosso, "High-resolution bottomloss estimation using the ambient-noise vertical coherence function," J. Acoust. Soc. Am. 137, 481-491 (2015).

${ }^{12}$ M. Siderius, L. Muzi, C. H. Harrison, and P. Nielsen, "Synthetic array processing of ocean ambient noise for higher resolution seabed bottom loss estimation," J. Acoust. Soc. Am. 133, EL149-EL155 (2013).

${ }^{13}$ J. E. Quijano, S. E. Dosso, M. Siderius, and L. Muzi, "Coherence extrapolation for underwater ambient noise," J. Acoust. Soc. Am. 135, EL318-EL323 (2014).

${ }^{14}$ P. L. Nielsen, M. Siderius, and L. Muzi, "Performance assessment of a short hydrophone array for seabed characterization using natural-made ambient noise," J. Acoust. Soc. Am. 136, 2155 (2014).

${ }^{15}$ C. H. Harrison, "Sub-bottom profiling using ocean ambient noise," J. Acoust. Soc. Am. 115, 1505-1515 (2004).

${ }^{16}$ J. E. Quijano, S. E. Dosso, J. Dettmer, L. M. Zurk, M. Siderius, and C. H. Harrison, "Bayesian geoacoustic inversion using wind-driven ambient noise," J. Acoust. Soc. Am. 131, 2658-2667 (2012).

${ }^{17}$ C. H. Harrison and M. Siderius, "Bottom profiling by correlating beam-steered noise sequences," J. Acoust. Soc. Am. 123, 1282-1296 (2008).

${ }^{18}$ G. B. Deane, M. J. Buckingham, and T. Tindle, "Vertical coherence of ambient noise in shallow water overlying a fluid seabed," J. Acoust. Soc. Am. 102, 3413-3424 (1997). 
${ }^{19}$ W. S. Liggett and M. J. Jacobson, "Noise covariance and vertical directivity in a deep ocean," J. Acoust. Soc. Am. 39, 280-288 (1966).

${ }^{20}$ D. R. Barclay and M. J. Buckingham, "Depth dependence of wind-driven, broadband ambient noise in the Philippine Sea," J. Acoust. Soc. Am. 133, 62-71 (2013)

${ }^{21}$ M. J. Buckingham, "A theoretical model of ambient noise in a low-loss, shallow water channel," J. Acoust. Soc. Am. 67, 1186-1192 (1980).

${ }^{22}$ B. F. Cron and C. H. Sherman, "Spatial-correlation functions for various noise models," J. Acoust. Soc. Am. 34, 1732-1736 (1962).

${ }^{23} \mathrm{H}$. Cox, "Spatial correlation in arbitrary noise fields with application to ambient sea noise," J. Acoust. Soc. Am. 54, 1289-1301 (1973).

${ }^{24} \mathrm{~W}$. A. Kuperman and F. Ingenito, "Spatial correlation of surface generated noise in a stratified ocean," J. Acoust. Soc. Am. 67, 1988-1996 (1980).
${ }^{25} \mathrm{H}$. Nakahara, "A systematic study of theoretical relations between spatial correlation and Green's function in one-, two- and three-dimensional random scalar wavefields,” Geophys. J. Int. 167, 1097-1105 (2006).

${ }^{26}$ S. C. Walker and M. J. Buckingham, "Spatial coherence and cross correlation of three-dimensional ambient noise fields in the ocean," J. Acoust. Soc. Am. 131, 1079-1086 (2012).

${ }^{27}$ C. H. Harrison, "Formulas for ambient noise level and coherence," J. Acoust. Soc. Am. 99, 2055-2066 (1996).

${ }^{28} \mathrm{C}$. H. Harrison, "Noise directionality for surface sources in rangedependent environments," J. Acoust. Soc. Am. 102, 2655-2662 (1997).

${ }^{29} \mathrm{H}$. Schmidt, OASES Version 3.1, User Guide and Reference Manual (Massachusetts Institute of Technology, Cambridge, MA, 2004). 